\title{
Acute Pancreatitis Associated with Mycoplasma pneumoniae: A Case Report of Missed Diagnosis
}

\author{
Abdulla Al-Abassi \\ Department of Surgery, Saqr Hospital, Ras Al-Khaimah, United Arab Emirates
}

\section{Key Words}

Acute pancreatitis - Extrapulmonary manifestations . Mycoplasma pneumoniae

\begin{abstract}
Objective: Mycoplasma pneumoniae is responsible for approximately $20 \%$ of community-acquired pneumonia. A wide variety of extrapulmonary manifestations related to $M$. pneumoniae infection can occur. The diagnosis of M. pneumoniae and its association with acute pancreatitis is briefly reviewed. Clinical Presentation: We describe the case of a 9-year-old boy with clinical features of acute abdomen. Intervention: A perforated appendix was suspected and the patient was accordingly operated on. The clinical, radiological and serological findings that led to the proper diagnosis are described. Conclusion: The case is presented to help increasing the awareness of M. pneumoniae infection as a potential cause of acute pancreatitis in children.
\end{abstract}

Copyright $\odot 2002$ S. Karger AG, Basel

\section{Introduction}

Mycoplasma pneumoniae is an important and treatable cause of community-acquired atypical pneumonia in children. It accounts for as many as $20 \%$ of all cases of community-acquired pneumonia in the general population $[1,2]$. The clinical course of pneumonia due to M. pneumoniae is typically mild and self-limited [3]. There are, however, several case reports of acute pancreatitis and other severe complications following this illness with considerable morbidity and mortality [1-5]. In some of these cases respiratory manifestations may be absent [2].

To our knowledge, only a few proven cases of acute pancreatitis in association with M. pneumoniae have been reported [6].

\section{Case Report}

A previously healthy 9-year-old boy presented to his primary care physician with mild fever, dry cough, anorexia, mild central abdominal pain and occasional vomiting in relation to food. He was treated for upper respiratory tract infection with appropriate doses of Augmentin (amoxicillin; clavulanate potassium) and paracetamol as required. On the 4th day of his illness, abdominal pain was persis-

\begin{tabular}{ll}
\hline KARGER & ( 2002 S. Karger AG, Basel \\
Fax +4161306 1234 & 1011-7571/02/0112-0112\$18.50/0 \\
$\begin{array}{l}\text { E-Mail karger@karger.ch } \\
\text { www.karger.com }\end{array}$ & $\begin{array}{l}\text { Accessible online at: } \\
\text { www.karger.com/journals/mpp }\end{array}$
\end{tabular}

Dr. Abdulla Ahmed Al-Abassi

PO Box 3405, Ras Al-Khaimah Branch

Ras Al-Khaimah (United Arab Emirates)

Tel. +971 72361157 , Fax +971 72222760

E-Mail alabassi@yahoo.com 


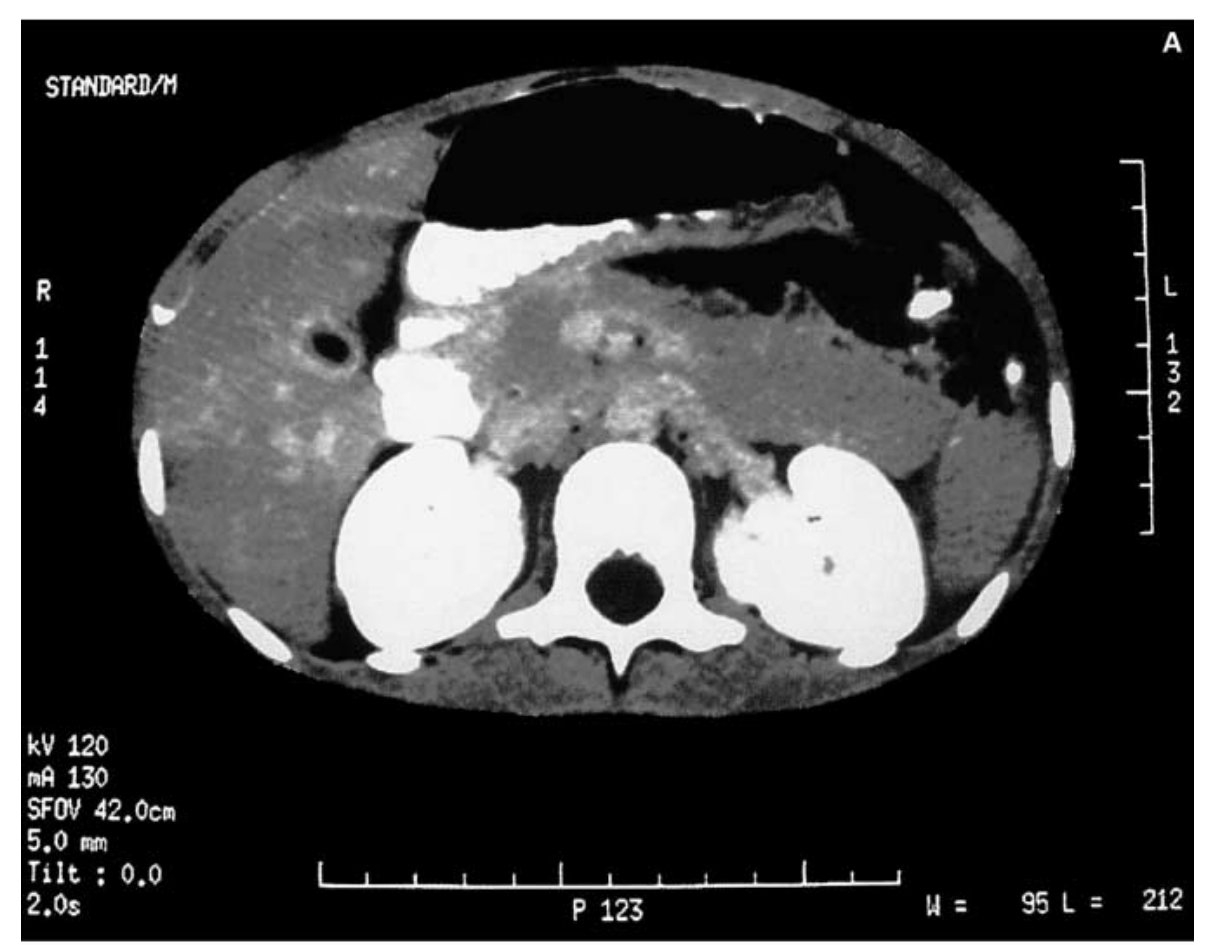

Fig. 1. Acute pancreatitis associated with M. pneumoniae infection. Enhanced computed tomography of the abdomen with oral and intravenous contrast. A Pancreatic enlargement with irregular contour and areas of decreased attenuation (phlegmon), notably at its head. B Extrapancreatic fluid collection (phlegmon) with prominent thickening of the root of the mesentery and adjacent parts of jejunum and ileum.

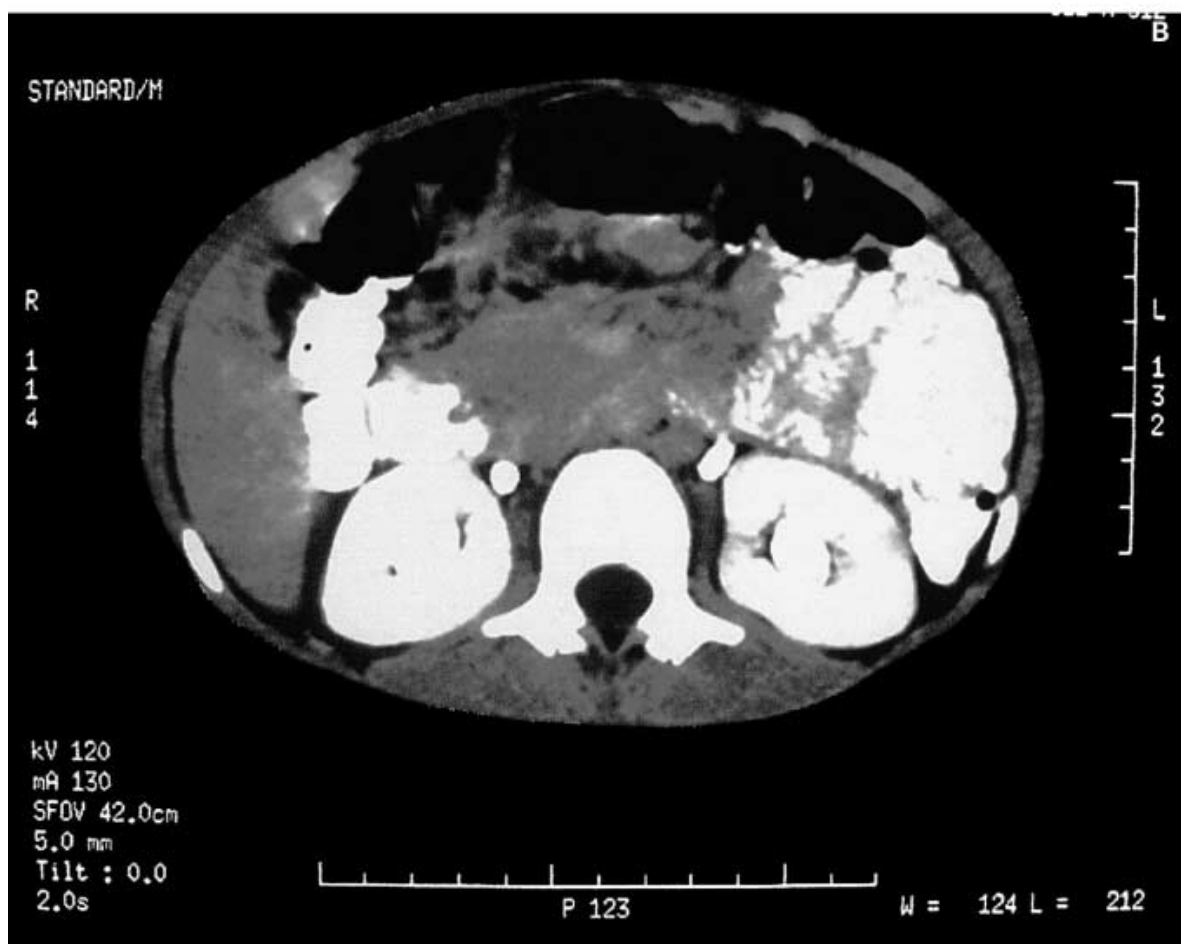

tent, severe, and more prominent on the right side of the abdomen. He was referred to our surgical department to exclude appendicitis. On admission, he was mildly dehydrated, and his temperature was $38.8^{\circ} \mathrm{C}$, pulse rate was 128 beats/min, and blood pressure was $110 /$ $70 \mathrm{~mm} \mathrm{Hg}$. Few discrete submandibular and upper cervical lymph nodes were palpable. His abdomen was not distended; there was ten- derness and guarding all over the abdomen, maximally on the right side. Mild rebound tenderness was also present on percussion. His bowel sounds were absent. The findings on general physical examination were otherwise normal. Complete blood count revealed hemoglobin of $16.2 \mathrm{~g} / \mathrm{dl}$, a white cell count of $11.14 \times 10^{3} / \mu 1$, and a hematocrit of $47.9 \%$. Serum creatinin, albumin, bilirubin, and liver 
enzymes were normal. The chest X-ray was normal, and abdominal X-ray film showed multiple air-fluid levels (suggestive of paralytic ileus). Abdominal ultrasonography revealed a free intraperitoneal fluid collection in the upper peritoneal recesses, iliac fossae, and pelvis. The pancreas could not be visualized because of small bowel gasses. Other intra-abdominal solid organs were normal.

Provisional diagnosis of acute abdomen was made, the most probable cause of which was thought to be a perforated appendix. The patient was operated on under general anesthesia through an incision centered at the right McBurney point. This corresponds to a point at the junction of the middle and outer thirds of a line joining the umbilicus to the right anterior superior iliac spine.

Approximately $400 \mathrm{ml}$ of serous fluid was aspirated, a sample of which was taken for biochemical analysis and culture. The appendix looked normal. Few enlarged terminal ileal lymph nodes were noted. The appendix and a terminal ileal mesenteric lymph node were excised and sent for histopathological examination.

Piperacillin, $1 \mathrm{~g}$ intravenously, was started with induction of anesthesia and continued postoperatively 6-hourly for 5 days.

Acute pancreatitis was suspected at operation on the basis of clear ascitic fluid in the background of normal liver and kidney function tests. This was confirmed by a high peritoneal fluid amylase of 4,410 units/1. After surgery, serum amylase was 525 units/1, and lipid profile was normal. Computed tomography of the abdomen showed pancreatic enlargement with areas of decreased attenuation (phlegmon), notably at its head (fig. 1A). Extrapancreatic fluid collection was also noted. The root of the mesentery, the proximal jejunal and ileal loops were prominently thickened (fig. 1B). The overall picture was that of severe acute pancreatitis (CT grading III-IV) [7].

Histopathological examination revealed a normal appendix and features of reactive tissue hyperplasia with sinus histiocytosis of the excised mesenteric lymph node. Direct culture of a peritoneal fluid sample did not grow bacteria.

Follow-up computed tomography of the abdomen 6 weeks later showed marked improvement with regard to phlegmon collection, with no evidence of pseudocyst formation.

Serological screening revealed nonsignificant titers for influenza A and B, adenoviurs, Q-fever and chlamydia antibodies. Measles and mumps IgM-specific antibodies were not detectable. $M$. pneumoniae antibodies were detected by complement fixation test. The antibody titer was 1:80 (acute infection is suspected if the titer is $>1: 20$ ). Repeated serological test with particle agglutination test for $M$. pneumoniae 6 weeks later revealed a fourfold increase in antibody titer indicating an acute $M$. pneumoniae infection.

Postoperatively, the patient showed sustained improvement. He was discharged on the 12 th postoperative day. He had no further symptoms or complications on regular follow-up over the subsequent 6 months.

\section{Discussion}

Acute pancreatitis is a relatively uncommon disease in children. In contrast to adults in whom $80 \%$ of cases of acute pancreatitis are associated with alcoholism and biliary disease, the etiology in children is diverse. Lerner et al. [8] summarized the etiological causes of acute pancreatitis in children as: idiopathic $22 \%$, trauma $20 \%$, infec- tion $15 \%$, biliary tract disease $14 \%$, drugs $13 \%$, miscellaneous $11 \%$ and congenital anomalies $5 \%$.

M. pneumoniae has never been identified in pancreatic specimens, and the association of $M$. pneumoniae with acute pancreatitis is based on isolated case reports and serological surveys of patients with acute pancreatitis [6]. This is partly because $M$. pneumoniae are very tiny organisms $(<350 \mu \mathrm{m}$ in length) and are not visible at light microscopy. Unlike other Mycoplasma species, they grow more slowly and take 1-3 weeks before colonies can be detected [2]. Besides, there is deficient knowledge of the epidemiology and extrapulmonary manifestations of their infection, so that the diagnosis often is not considered [9]. A definite diagnosis of pancreatitis is based on evidence of pancreatitis at surgery of radiological evidence of pancreatitis by ultrasound and/or computed tomography, while a probable diagnosis of pancreatitis is based on characteristic symptoms and a threefold increase in amylase. A definite diagnosis of the etiological agent is based on identifying the organism in pancreatic tissue obtained by open or percutaneous biopsy, either by staining or culture, while probable evidence of the infection is determined by detection of the etiological agent in blood or pancreatic fluid and/or serological diagnosis based on a fourfold rise in antibody titer [6]. Based on this classification, our case proved to be a definite diagnosis of acute pancreatitis and a probable evidence of Mycoplasma infection being the etiological agent. Taking into consideration that other possible causes of acute pancreatitis have been excluded, our case clearly demonstrates the strong association between M. pneumoniae infection and acute pancreatitis.

The exact mechanism by which pneumoniae induce pancreatitis is not well established. Possible mechanisms include ascension of Mycoplasma organisms up the pancreatic duct from small bowel or biliary tree, seeding via the blood or lymphatic system, organ-specific toxin production, and autoimmune response to Mycoplasma infection $[1,6]$.

Appropriate antibiotic therapy for M. pneumoniae infection, usually erythromycin, clarithromycin, azithromycin or tetracycline, can help prevent spread of the infection to contacts, and limit the course of the respiratory disease in the patient [2]. In most cases, extrapulmonary manifestations are preceded by respiratory symptoms, and treatment of pulmonary disease seems to have little effect on subsequent nonrespiratory disease [2]. 


\section{Conclusion}

The laboratory results and clinical findings for our patient show that he had a recent $M$. pneumoniae infection and a concurrent episode of acute pancreatitis. No coincidental causes of acute pancreatitis were evident. Viral infections most commonly associated with acute pancreatitis have not been detected on serological screening. The findings in this case suggest that $M$. pneumoniae infection induced acute pancreatitis. We therefore believe that M.pneumoniae infection should be considered among the possible causes of acute pancreatitis. It may also be considered as a rare possible cause of acute abdomen in children.

\section{Acknowledgment}

The author gratefully appreciates the cooperation of Dr. Mohammed Emad, MD, Head of Radiology Department, Dr. Farook Awwad, MD, Director of Laboratories Department and Ms. Deirdre McEnery, AIMLT, Senior Charge Technician in Serology Laboratory, Saqr Hospital, Ras Al-Khaimah, United Arab Emirates.

\section{References}

1 Berger RP, Wadowksy RM: Rhabdomyolysis associated with infection by Mycoplasma pneumoniae: A case report. Pediatrics 2000;105: 433-436.

2 John SD, Ramanathan J, Swischuk LE: Spectrum of clinical and radiographic findings in paediatric Mycoplasma pneumonia. Radiographics 2000;21:121-131.

3 Kim CK, Chung CY, Kim JS, Kim WS, Park Y, Koh YY: Late abnormal findings on highresolution computed tomography after $\mathrm{Myco}$ plasma pneumonia. Pediatrics 2000;105:372378.
4 Dionisio D, Valassina M, Mata S, Rossetti R, Vivarelli A, Esperti FC, Benvenuti M, Catalani C, Uberti M: Encephalitis caused directly by Mycoplasma pneumoniae. Scand J Infect Dis 1999;31:506-509.

5 Perez C, Gurtubay F, Martinez-Ibanez F, Quesada P, Maravi E: More on polymyositis with Mycoplasma pneumoniae infection. Scand J Rheumatol 1999;28:125.

6 Parenti DM, Steinberg W, Kang P: Infectious causes of acute pancreatitis. Pancreas 1996;13: 356-371.
7 Balthazar EJ, Robinson DL, Megibow AJ, Ranson JH: Acute pancreatitis: Value of CT in establishing prognosis. Radiology 1990;174: 331-336.

8 Lerner A, Branski D, Lebenthal E: Pancreatic diseases in children. Pediatr Clin North Am 1996;43:125-156.

9 Baum SG: Mycoplasma pneumoniae and atypical pneumonia; in Mandell GL (ed): Principles and Practice of Infectious Diseases. Edinburgh, Churchill Livingstone, 2000, pp 2018-2025. 